\title{
Analysis of Machine-made Sand Parameters in Shaanxi
}

\author{
Zhangjun Chen, Kangjian Yu, Donghui Miao*, Yuliang Ke, Bobo Liu \\ Shaanxi Kezhijie New Materials Co. LTD, Xian 710000, China
}

\begin{abstract}
Machine-made sand was significantly different from natural sand in terms of particle gradation, morphology, composition and structure. The concrete composed of mechanical sand and natural sand was greatly influenced by the physical and chemical properties of sand and gravel in terms of its microscopic appearance and macroscopic properties. Therefore, this paper aimed to systematically analyze the big data of machine-made sand performance testing, and focus on the fluctuation of the fineness modulus, stone powder content, and other indicators of machine-made sand particles in various regions of Shaanxi, and solve measures were further discussed.
\end{abstract}

\section{Introduction}

Concrete is one of the key materials required for engineering construction, and it is also the largest and most widely used engineering material in the world, which plays a significant role in the construction of bridges, nuclear power, high-rise buildings, etc[1]. Sand and gravel aggregate is an indispensable and key part of concrete production, accounting for about $70 \%$ to $80 \%$ of concrete quality[2]. Construction projects require a large amount of sand and gravel resources each year, but the self-sufficiency rate of natural sand is getting lower and lower, and it is difficult to meet the needs of current engineering construction, which largely restricts the development of related industries. At the same time, sand and gravel aggregates are non-renewable and unsuitable for long-distance transportation, which has further led to a prominent contradiction between the supply and demand of sand and gravel in some areas. As a substitute for natural sand, machine-made sand can relieve the current shortage of natural sand resources. In view of this problem, in 2016, Shannxi Province issued the "Technical Regulations for the Production and Application of Machine-made Sand" (DBJ61T 1372017), which further standardized the production methods of Shannxi Province's machine-made sand to meet the relevant requirements of ready-mixed concrete and ready-mixed mortar. However, machine-made sand is significantly different from natural sand in terms of particle gradation, morphology, and composition structure. Because the concrete composed of machinemade sand and natural sand is affected by the physical and chemical properties of sand and stone in terms of microscopic morphology and macroscopic properties. Therefore, this article takes the machine-made sand in Shaanxi as the starting point, and focuses on the fluctuations of the grain gradation, fineness modulus, stone powder content and other indicators of the machine-made sand in Shaanxi. The measures is discussed to form a concrete application technical guide for the mechanism of mixing station, which can guide the mixing station to carry out production control and effectively reduce the occurrence of concrete quality accidents.

\section{Experimental}

In this experiment, machine-made sand produced by different enterprises in Shaanxi and surrounding areas were selected as raw materials for analysis. Samples were numbered 1 to 10 , and their physical and chemical properties were tested with reference to GB/T 1484-2011.

\section{Results and Discussion}

\subsection{Analysis of stone powder content}

In order to analyze the stone powder content in different machine-made sands, this experiment selected samples from 10 machine-made sand manufacturers in Xi'an and surrounding areas. The stone powder content in different machine-made sands was shown in Figure 1. It could be seen from Figure 1 that the stone powder content in sample 3 was the lowest at $1.9 \%$, and the stone powder content in sample 10 was the highest at $61.8 \%$. The stone powder content in the remaining samples was basically distributed between $5 \%$ and $20 \%$, and the overall fluctuation was large. The reasons for the difference in stone powder content of machine-made sands in different regions could be summarized as follows: (1) Different sources of raw materials for machine-made sand. Generally speaking, machine-made sand was mainly made of base material (granite, river pebbles, basalt, etc.) crushed and screened. The overall quality of this kind of machine-made sand was relatively good. The purity of machine-made sand after treatment was

\footnotetext{
a Corresponding author: Donghui Miao @ cel11989@163.com
} 
relatively high, the stone powder content was also relatively low. In addition to, the machine-made sand prepared from the base material, iron tailings waste rock and construction solid waste could be processed by crushing and screening equipment, which had a wide range of sources and complex composition, and the prepared sand had mud content, the content of stone powder was relatively high, especially the iron tailing waste rock machine-made sand, which had higher stone powder content. Sample 10 was a machine-made sand processed by iron tailing waste rock, and its stone powder content was much higher than other machines sand samples. (3) There were differences in the production process of machine-made sand[3]. The current production processes of machine-made sand mainly included wet and dry production processes. There were also significant differences in quality and stone powder content of machine-made sand prepared by different production processes. The cleanliness of machine-made sand produced by wet method was relatively high, the stone powder content was lower, and the color appearance was similar to natural sand. It was currently a machine-made sand production method favored by enterprises. The appearance of machine-made sand produced by dry method was obviously different from that of natural sand. When the stone powder content of raw machine-made sand produced by dry method was about $10 \%$, it looked like stone powder (or soil). If the post-processing was improper, the stone powder content of machine-made sand and gravel powder was significantly higher than that of wetprocessed machine-made sand. In view of the advantages and disadvantages of dry and wet production, the combined dry-wet production process was currently the preferred production process adopted by some manufacturers.

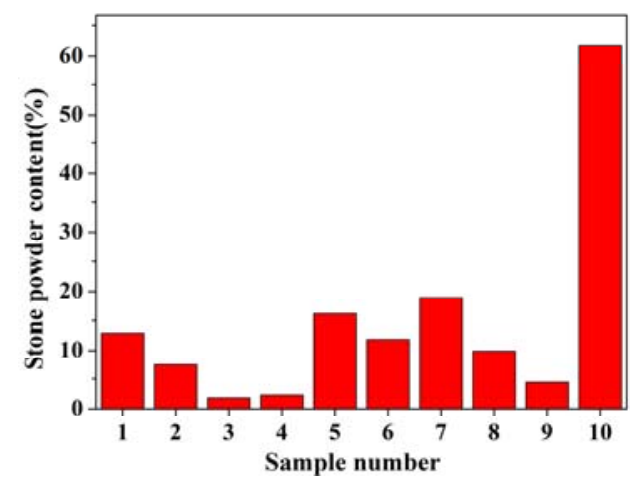

Figure 1. Powder content of different mechanism sand

Zhu et al [4]studied that the effect of high-quality machine-made sand-gravel powder on the performance of C30 concrete. The results showed that appropriate amount of stone powder could improve the gradation of machine-made sand and filled the gaps between particles. With the increase of stone powder content, the workability, compressive strength, air permeability and frost resistance of machine-made sand concrete would be improved on the basis of the increase of stone powder content. As shown in Figure 2, the optimum mechanism sand powder content of C30 concrete ranged from $9 \%$ to
15\%. Geng Quan et al[5] also conducted a similar study, and the results showed that when the stone powder content was $8 \%$ to $15 \%$, as the stone powder content increase, the density of the machine-made sand concrete interface transition zone increased, the number of voids decreased, and the crack size changed. At the same time, appropriately increasing the stone powder content could increase the anti-chloride ion permeability of the machine-made sand concrete. Conversely, when the stone powder content exceeded $15 \%$, anti-chloride ion permeability of the machine-made sand concrete decreased. Therefore, the stone powder content should be strictly controlled in the production process of machine-made sand. The possible control measures were as follows: (1) The reasonable coordination of multilayer mesh [6]. If the mesh was not coordinated, the mesh efficiency would decrease, and the materials entering the mesh will be difficult to screen through, and the qualified sand will enter the sand making machine again, and the content of stone powder would increase due to the secondary crushing, resulted in increase of stone powder content. (2) Control the source of raw materials for machine-made sand base materials. In view of the current situation of extensive sources of machinemade sand base materials, machine-made sand manufacturers should establish their own quality management system according to the actual situation and grasp the source classification, especially, when the construction solid waste was converted into recycled sand and gravel aggregate, material classification should be classified, and different production processes should be adopted for materials with different characteristics, and if necessary, secondary treatment processes should be adopted. (3) Extensiveness of testing and sampling of stone powder content. Due to the phenomenon of fine separation and segregation of finished machine-made sand on the belt conveyor, if the sample was not extensively sampled or the sample was not fully mixed before the test was taken, which caused that the sample would be distorted, and would affect the accuracy of the test result. Therefore, sampling must be taken from the whole section of the belt conveyor, at least three times samplings were required. After multiple samplings, all samples were thoroughly mixed in the stirring device and then the stone powder content would be tested.

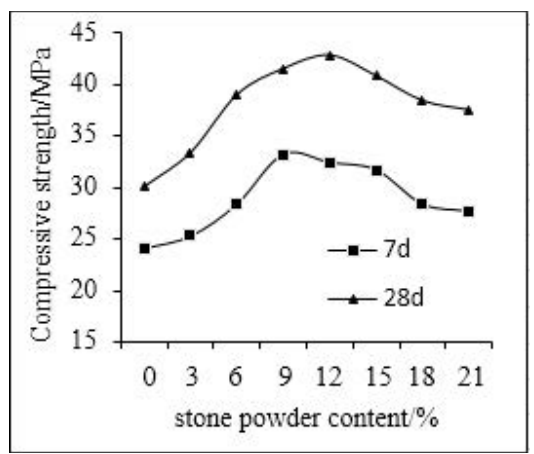

Figure 2. Compressive strength of $\mathrm{C} 30$ concrete $^{[4]}$ 


\subsection{Analysis of mud content}

The mud content in different machine-made sand was shown in Figure 3. It could be seen from Figure 3 that the mud content in sample 1 was the highest at $1.9 \%$, and the mud content in sample 3 was the lowest at $0.4 \%$. The mud content in the remaining samples was basically distributed between $1 \%$ and $8 \%$, the average value was $3.8 \%$. Shaanxi Province stipulated that the mud content of natural rock machine-made sand and iron tailing waste rock machine-made sand should meet type $\mathrm{I}=0.0 \%$, type $\mathrm{II} \leqslant 0.5 \%$, type $\mathrm{III} \leqslant 1.0 \%$; in the fine grain sand of iron tailings, the mud content should not be greater than $1.0 \%$; for the reclaimed machine-made sand, the mud content should meet: type I $<0.5 \%$, type II $<1.0 \%$, and type III $<2.0 \%$. According to the analysis of this standard, we found that the qualified rate of the mud content of machine-made sand in Shaanxi and surrounding areas was about $40 \%$. In other words, the mud content of the machine-made sand produced by most manufacturers was much higher than the regional standard. The quality of the whole mechanism sand industry was not uniform, and the development situation was not optimistic, which maby have a serious impact on the performance of machine-made sand concrete. The mud content in the machine-made sand mainly affected the performance of the transition zone of the concrete interface, the water reducing effect of the water reducing agent, and the strength of the concrete. Excessive mud content would weaken the bonding performance of the concrete interface transition zone, increased the porosity and cracks, and the microstructure of the concrete would become more fragile, thereby affecting its mechanical properties. As shown in Figure 4, we could find that with the mud content increases, the $28 \mathrm{~d}$ compressive strengths of concrete gradually decreased. when the mud content was low, the microstructure of concrete was relatively compact, the number of pores was less, the width of cracks was narrower, and the compressive strength was larger. As the mud content increase, the concrete structure became loose and porous, the number of pores increased, the size of the transition zone of the concrete interface became larger, and the compressive strength decreased[7]. In addition, there are "selective adsorption"and "competitive adsorption" behaviors between the clay in the machine-made sand and the polycarboxylic acid water reducing agent. Therefore, the presence of soil particles reduced the effective adsorption of polycarboxylic acid molecules on the surface of cement particles, which reduced the water reducing effect of polycarboxylic acid. To suppress the adverse effects of mud, the following measures could be taken:(1) Design a new type of anti-sludge polycarboxylic acid based on the molecular level, and introduce anti-sludge groups into the polycarboxylic acid molecule by modifying the existing polycarboxylic acid molecular structure, such as phosphoric acid groups, silanol groups, nitrogen-containing functional groups[8], which could also synthesize polycarboxylic acids with long side chains without polyethylene glycol, and design and synthesize polycarboxylic acids with a star-like structure, increase the steric hindrance to reduce the occurrence of soil intercalation adsorption [9] and reduce its impact on the efficiency of the water reducer. (2) Before the master batch was crushed, master batch could be cleaned by ultrasonic cleaning, stirring cleaning, increasd the cleaning water pressure, multi-stage immersion cleaning and other methods to clean the particles and sludge on the surface of the material, thereby reduced the mud content in the machine-made sand.

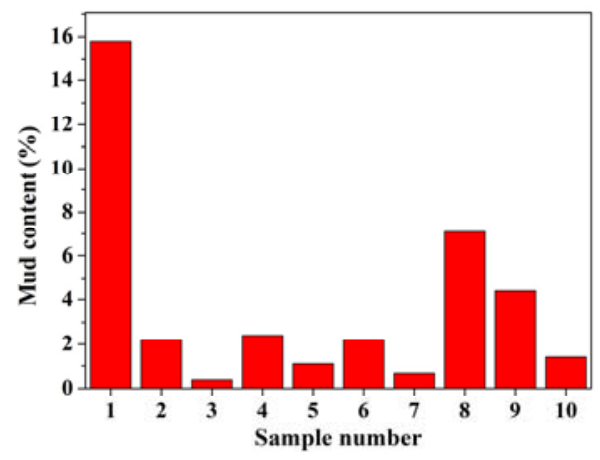

Figure 3. mud content of different mechanism sand

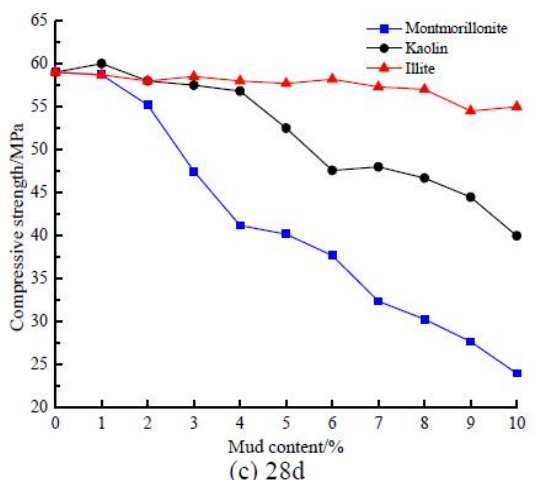

Figure 4. Compressive strength-mud content curves $^{[7]}$

\subsection{Fineness modulus analysis}

The fineness modulus in different machine-made sand was shown in Figure 5. It could be seen from Figure 5 that the machine-made sand in Shaanxi was relatively coarse, which the sand in zone I accounted for $40 \%$, the sand in zone II accounted for $50 \%$, the sand in zone III accounted for $10 \%$, and the number of machine-made sand samples with fineness modulus of more than 3.0 accounted for $30 \%$. According to JGJ 52 "Standard for Quality and Inspection Methods of Sand and Stone for Ordinary Concrete", "medium sand should be selected for the preparation of pumped concrete", which may be approximately considered inappropriate to prepare pumped concrete. According to JGJ 52 "Standard for Quality and Inspection Methods of Sand and Stone Used for Ordinary Concrete", "Section 2 medium sand should be selected for the preparation of pumped concrete", collecting 10 kinds of machine-made sand samples had 4 kinds of machine-made sand particle gradation distribution in zone I, the proportion was up to $40 \%$, and there were three types with a fineness modulus exceeding 3.0, which accounted for $30 \%$, indicating that the existing machine-made sand samples in our province 
still had the problems of poor particle gradation and high fineness modulus. Zhuang et al [10] studied the influence of the fineness modulus of machine-made sand on the working performance of concrete, and the results showed that under the premise of certain sand ratio and machine-made sand powder content, the fineness modulus of machine-made sand mainly effected the water absorption of the particle surface. bulk density, concrete strength. Therefore, in actual engineering applications, the production process of machine-made sand should be adjusted so that the particle gradation of machine-made sand tends to be excellent to ensure the excellent working performance of machine-made sand concrete.

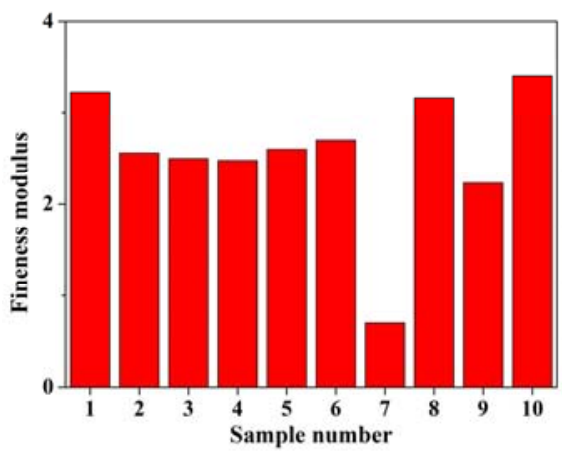

Figure 5. Mud content of different mechanism sand

\subsection{Analysis of apparent density, bulk density and porosity analysis}

Table 1. Apparent density, bulk density, porosity of different mechanism sand

\begin{tabular}{cccc}
\hline \multirow{2}{*}{$\begin{array}{c}\text { Sample } \\
\text { number }\end{array}$} & \multicolumn{3}{c}{ Parameter of mechanism sand } \\
\cline { 2 - 4 } & $\begin{array}{c}\text { Apparent } \\
\text { density } / \mathrm{kg} / \mathrm{m}^{3}\end{array}$ & $\begin{array}{c}\text { bulk density } \\
/ \mathrm{kg} / \mathrm{m}^{3}\end{array}$ & porosity $/ \%$ \\
\hline 1 & 2720 & 1690 & 38 \\
2 & 2600 & 1680 & 35 \\
3 & 2770 & 1880 & 32 \\
4 & 2570 & 1810 & 30 \\
5 & 2700 & 1760 & 35 \\
6 & 2850 & 1700 & 40 \\
7 & 2650 & 1660 & 37 \\
8 & 2760 & 1650 & 40 \\
9 & 2660 & 1690 & 36 \\
10 & 2690 & 1690 & 37 \\
\hline
\end{tabular}

Table 1 showed the apparent density, bulk density and porosity of ten kinds of machine-made sand samples in Shaanxi. According to GB/T 14684 "Sand for Construction", the apparent density of sand was not less than $2500 \mathrm{~kg} / \mathrm{m} 3$, the bulk density was not less than $1400 \mathrm{~kg} / \mathrm{m} 3$, and the porosity was not more than $44 \%$. The test results showed that ten kinds of the apparent density bulk density of machine-made sand samples reached the specified value of GB/T 14684 "Sand for Construction", the porosity of ten kinds of machinemade sand samples were all lower than the specified value of GB/T 14684 "Sand for Construction". From the above analysis, we believed that the apparent density, bulk density, and porosity of the machine-made sand in Shaanxi were basically in line with the national standard values, and some parameters of machine-made sand were better than the national standard values.

\subsection{Analysis of MB value}

Figure 6 showed the MB value analysis of 10 kinds of machine-made sand samples in Shaanxi. It could be seen from Figure 6 that MB value of two kinds of machinemade sand was less than 1.4 , accounting for $20 \%$, and $\mathrm{MB}$ value of eight kinds of machine-made sand was greater than 1.4 , accounting for $80 \%$. Among them, the $\mathrm{MB}$ value was the largest sample 3 , and the $\mathrm{MB}$ value reached to 4.8. Generally speaking, the $\mathrm{MB}$ value of the samples provided by most of the machine-made sand production enterprises in Shaanxi did not conform to the "Sand for Construction" GB 14684-2011, enterprises should strengthen the control of mechanism sand MB value, to avoid concrete quality accidents caused by excessive mechanism sand MB value.

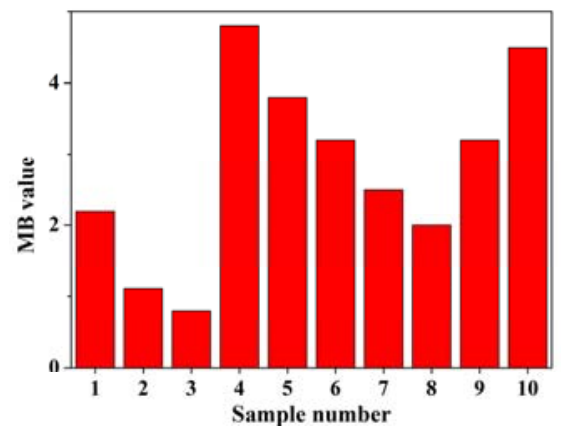

Figure 6. MB value of different mechanism sand

\section{Conclusions}

The main quality problems of mechanism sand in Shaanxi were high fineness modulus, poor particle gradation, and large fluctuation range of $\mathrm{MB}$ value exceeding national standard and quality unstable physical performance index. Therefore, mechanism sand production enterprises in Shaanxi should improve production process, optimize mechanism sand particle gradation, and reduce mechanism sand $\mathrm{MB}$ value to make it conform to national standard.

\section{References}

1. M. Chao. Guangdong building materials, 36 (2020).

2. F. Pei Ran. Cement engineering, 5(2020).

3. L Yong, L Yu. Fujian Architecture, 138(2019).

4. Zhu Y, Wen C, Chen J. MS\&E, 371(2018).

5. G. Quan, C, Li Bing. Shaanxi Architecture, 43(2017).

6. F. Hai Long. Equipment maintenance technology, $7(2020)$.

7. Q. Wang. Annales de Chimi - Science des Matériaux, 43(2019).

8. L. Lei, Plank J. Cement and Concrete Research, 42 (2012). 
9. C. Juan. Jiangxi building materials, 1(2020).

10. Z. Shao Mu. Journal of Guangdong Polytechnic of Communications, 2 (2019). 